\title{
Biophysical characterization of MDR cell lines reveals the cytoplasm is critical in determining drug sensitivity
}

\author{
Fatima H. Labeed ${ }^{1}$ \\ Michael P. Hughes ${ }^{1}$ \\ Hilary Thomas ${ }^{2}$ \\ Helen M. Coley ${ }^{2}$
}

${ }^{1}$ Centre of Biomedical Engineering, School of Engineering, University of Surrey, Guildford, Surrey GU2 7XH, United Kingdom

${ }^{2}$ Division of Oncology, Postgraduate Medical School, School, University of Surrey, Guildford, Surrey GU2 7WG, United Kingdom

\author{
*Corresponding author \\ E-mail: <h.coley@surrey.ac.uk> \\ Phone: 0044-1483-688617 \\ Fax: 0044-1483-688604
}

Running title: Biophysical properties of MDR cells

Key words: Dielectrophoresis, P-gp, MDR, drug sensitivity 


\section{Summary}

Dielectrophoresis (DEP) was used to examine a panel of MCF-7 cell lines comprising MDR derivatives: MCF-7TaxR (paclitaxel -resistant, P-glycoprotein (P-gp) positive), MCF-7DoxR (doxorubicin-resistant P-gp negative) plus MCF-7MDR1 (MDR1 transfected, P-gp positive). MCF-7DoxR and MCF-7MDRl were broadly crossresistant to natural product anticancer agents, whereas MCF-7TaxR cells were not, contrary to P-gp expression. Whilst DEP revealed modest membrane changes in MDR sublines, we saw significant changes in their cytoplasmic conductivity: MCF7TaxR < MCF-7<MCF-7MDR1 < MCF-7DoxR (range $0.14-0.40 S / m$ ). Cytoplasmic conductivity is affected by the movement of molecules e.g. as in intracellular trafficking MCF-7TaxR showed a reduced membrane potential, whereas MCF7DoxR and MCF-7MDR1 showed an increase. Thus, altered membrane potential is associated with an MDR phenotype, but in a complex manner. DEP data suggest a model whereby relative increases in cytoplasmic conductivity are correlated with MDR, whilst relative decreases equate with a sensitised phenotype e.g. MCF-7TaxR. Moreover, extent of anthracycline accumulation was inversely related to cytoplasmic conductivity. These data are representative of a model where drug sensitivity is associated with low ionic conductance (reduced cellular trafficking and ion transport) and substantial anthracycline accumulation. For classical MDR i.e. MCF-7MDRl, we saw the reverse picture. Thus, the drug resistance phenotypes of this panel of MCF-7 lines can be delineated by assessment of cytoplasmic biophysical properties using DEP. 


\section{Introduction}

It is a number of years since the first description of anticancer drug resistance [1], and since then many mechanisms have been described that attempt to explain the basis underlying this phenomenon. By far the most studied form of anticancer drug resistance is that of multidrug resistance (MDR), and findings from that research influenced to some extent research into MDR in microorganisms e.g. malaria and bacteria. Indeed, the cloning of $M D R$ genes overexpressed in MDR tumour cells resulted in the discovery of a large family of membrane located glycoproteins- the ATP-binding cassette (ABC) proteins. The large majority of studies have focused on MDR1 protein, also referred to as $\mathrm{P}$-glycoprotein (P-gp; recently renamed ABCB1).

Previous work by Wadkins and Roepe and others [2] went to some lengths to try to understand mechanisms that give rise to the MDR phenotype. For example, alterations in the membrane potential and also alkalinisation of the cytoplasm were shown to be features of P-gp expressing cells that could go some way towards explaining their drug resistant phenotype $[3 ; 4]$. In this paper we set out to explain on the basis of biophysical parameters, the drug sensitivities of a panel of novel MCF-7 drug resistant cell lines: MCF-7TaxR, MCF-7DoxR and MCF-7MDR1. In terms of their molecular characterisation, we show that MCF-7TaxR expresses P-gp but unusually it fails to demonstrate a broad cross-resistance (or multiple resistance) to natural product anticancer agents. The paclitaxel resistance in that cell line is due to P-gp overexpression as the modulating agent XR9576 (P-gp specific) completely reverses the resistance. On the other hand, MCF-7DoxR does not express P-gp, MRP1 (ABCC1) or BCRP (ABCG2) but shows broad cross-resistance to many natural product anticancer agents in line with an MDR phenotype. MCF-7MDRl is a classical 
MDR cell line showing both membrane P-gp overexpression and cross-resistance to natural product anticancer agents $[5 ; 6]$.

It has been shown that cells transfected with MDR cDNA without subsequent maintenance in the presence of chemotherapeutic agents can certainly mimic some of the features of the MDR phenotype [7]. However, the level of drug resistance seen in those cells is somewhat less than that seen in drug-selected lines with comparable levels of P-gp expression. Moreover, in terms of understanding the biophysical events associated with MDR it is clear that data obtained using 'pure' transfected models are somewhat at variance with those obtained using cells cultured in a drug containing environment. We have focused on the latter as our model system - which used a panel of cell lines including an MCF-7MDR1 transfected line but also maintained in a drug conditioned environment and, hence, not representative of a 'pure' transfection model. In terms of what is entirely relevant to the problem of clinical drug resistance and, hence, the ideal model has yet to be agreed upon by the cancer research community. Whilst it is imperative to define the effect of the MDRI gene expression itself on anticancer drug resistance, it is also important to understand those processes that bring about a drug-induced MDR phenotype. Acquired MDR phenotypes are well-documented phenomena and are highly relevant to the development of clinical drug resistance. Elegant studies such as those by Hoffman et al. [7] have been very important in helping us to understand a more precise role for $M D R 1$ in anticancer drug resistance. Notwithstanding, we propose that using cell line models with acquired drug resistant phenotypes obtained by growing up in a drug containing environment must bear some relation to the heavily pre-treated tumours that present with MDR in the clinic. Criticism of use of these types of cell line models 
has focused on the very high levels of drug resistance seen (sometimes in excess of 1000-fold) which have been deemed by many to be totally irrelevant to the problem of clinical drug resistance where 2-fold resistance to an anticancer agent will result in disease progression and kill the patient. We have used cell lines with relatively modest levels of drug resistance, maintained using drug doses relevant to clinically achievable blood levels.

Primarily, we have used the technique of dielectrophoresis (DEP) to understand the biophysical nature of the MCF-7 cell line panel, including the parental cells. DEP is a technique whereby the frequency-dependent behaviour of cells is determined in nonuniform electric fields, and from this information regarding the electrical properties of the cell can be inferred $[8 ; 9 ; 10]$. DEP and related techniques have been used to characterise a wide range of cellular processes in terms of these biophysical properties in cancer research [11-18]. Our studies have also incorporated a flow cytometric technique to measure membrane potential as an additional biophysical parameter to be considered in our assessment of the cell line panel. Previously we have shown that due to the nature of the putative drug efflux mechanism employed by MDR cells, dyebased methods of analysis (e.g. using DIOC5) to assess membrane potential can yield misleading results [19]. However, by combined use of an MDR modulating agent that corrects for defective DIOC5 accumulation that is recognised by $\mathrm{ABC}$ transporters, we can achieve a more realistic interpretation of membrane potential in an MDR cell line. We have also looked at the cellular accumulation of anthracyclines in our MCF-7 panel of lines, using fluorescence microscopy. These data have highlighted a close association between cytoplasmic conductivity and extent of drug accumulation in the present study. 
We demonstrate that there are significant changes in the cytoplasmic conductivity of all MDR cell lines relative to the parent line and also to each other. Interestingly, there are membrane changes measured using DEP seen for the paclitaxel-resistant line in terms of the membrane capacitance (Cspec) and not observed for either the doxorubicin-resistant or MDRI transfected MCF-7 lines. In addition, in line with its overall drug sensitivity profile, the MCF-7TaxR cell line is proficient in anthracycline accumulation, which is counterintuitive to the presence of membrane P-gp. Based on the present data, we can propose a model based on biophysical factors that can explain MDR whether in the absence or presence of Pgp or other ABC transporters. 


\section{Materials and Methods}

\section{Chemicals and reagents}

Paclitaxel (Taxol; obtained from Bristol Myers Squibb UK as a pharmacy preparation) and doxorubicin (Sigma Aldrich, Poole, UK) were dissolved in either sterile $0.9 \% \mathrm{NaCl}$ solution (paclitaxel) or in sterile distilled water (doxorubicin). They were stored frozen as stock solutions and were thawed prior to use. DIOC5 (obtained from Molecular Probes, Invitrogen, Paisley, UK) was made up as an aqueous stock solution and stored at $-20^{\circ} \mathrm{C}$ until use. XR9576 (kindly provided by Xenova PLC, Slough, UK) was made up as a stock solution in DMSO and stored frozen until use. PSC-833 (Valspodar; obtained from Novartis, Basel, Switzerland) was made up as a $5 \mathrm{mM}$ stock solution in ethanol, stored at $-20^{\circ} \mathrm{C}$ and then diluted accordingly for use in experiments.

\section{Cell culture}

All cell culture reagents were obtained from Sigma Aldrich (Poole, UK), unless stated otherwise. The MCF-7 human breast cancer cell line and its paclitaxel and doxorubicin resistant counterparts MCF-7TaxR and MCF-7DoxR, respectively were grown in 20mM HEPES modified Dulbecco's Modified Eagle Medium (DMEM) supplemented with 10\% heat-inactivated foetal calf serum (FCS), (Invitrogen, Paisley, UK) and $2 \mathrm{mM}$ L-glutamine. The cells were grown under standard incubation conditions at $37^{\circ} \mathrm{C}$. Both the drug resistant cell lines were derived in our laboratories by growing up in increasing step-wise concentrations of the inducing agent for a number of weeks until a stable resistance phenotype was achieved. MCF-7TaxR was maintained in the presence of $6 \mathrm{nM}$ paclitaxel, while MCF-7DoxR was maintained in the presence of $100 \mathrm{nM}$ doxorubicin. The MCF-7MDRl cell line, kindly provided by 
Professor Robert Clarke, Georgetown University, Washington DC, US, was stably transfected with the MDRI gene and maintained with selection pressure using 200ng/ml colchicine [20] The parental MCF-7 breast cancer cells were obtained from The European Collection of Cell Culture, Porton Down, Salisbury, UK (ECACC). All cultures were regularly checked for mycoplasma infection.

\section{Chemosensitivity testing}

MTT (3-(4,5-Dimethylthiazol-2-yl)-2,5-diphenyltetrazolium bromide) was used to titrate cell viability following drug treatment, as described by Mosmann [21]. MCF-7 cells were seeded at a density of the order of $2-4 \times 10^{4}$ cells $/ \mathrm{ml}$ (dependent upon doubling times of the particular cell lines) in supplemented tissue culture medium (see above). Cells were then dispensed into 96-well plates in volumes of $200 \mu \mathrm{L}$ and left to equilibrate for 24 hours at $37^{\circ} \mathrm{C}$ and $5 \% \mathrm{CO}_{2}$. Freshly thawed stock drug solutions were diluted in complete tissue culture medium and added at the appropriate concentration. Control wells containing cell suspension were supplemented with a similar volume of culture medium only. The MDR reversal agent XR9576 was used in combination with paclitaxel in the MCF-7TaxR line and in combination with doxorubicin in the MCF-7DoxR and MCF-7MDRl lines in order to ascertain whether resistance was attributable to P-gp. XR9576 was used at a final concentration of 100nM, in line with previous studies [22]. In addition, for some experiments we incorporated the use of PSC-833 at a final concentration of $5 \mu \mathrm{M}$ (as previously described in [23]) as a broad spectrum MDR modulator particularly to see if there was any modulation of resistance in the MCF-7DoxR cell line due to hitherto unidentified $\mathrm{ABC}$ transporters. 
Following 96-hour incubation, cultured cells were treated with $20 \mu \mathrm{L}$ of $5 \mathrm{mg} / \mathrm{mL}$ MTT in PBS (Sigma Aldrich, UK) for 4 hours at $37^{\circ} \mathrm{C}$. Wells were then aspirated dry and the tetrazolium crystals dissolved in $200 \mu \mathrm{L}$ of DMSO. Absorbance was read at 540nm using an automated ELISA plate reader. The results were expressed as $\mathrm{IC}_{50}$, i.e. the concentration of cytotoxic drug that reduces cell viability by $50 \%$ relative to the control (untreated cells).

\section{Western immunoblotting}

Crude cell membrane preparations were made from monolayers of cultured cells in the exponential phase of growth. Following detachment of cells using non-enzymatic cell dissociation fluid, cells were pelleted and washed twice in PBS and then resuspended in hypotonic lysis buffer containing detergent and protease inhibitors. 50 $\mu \mathrm{g}$ of membrane protein was loaded onto a $10 \%$ acrylamide SDS and resolved by SDS-PAGE. The presence of P-gp was detected with anti-P-gp rabbit polyclonal antibody, clone H241; MRP1 was detected using clone C-20 goat polyclonal antibody (both from Autogen Bioclear, Calne, UK) and BCRP was detected using clone Ab-1 mouse monoclonal antibody ( $\mathrm{CN}$ Biosciences, Beeston, Nottingham, UK). Visualisation was carried out using the appropriate species HRP conjugated with a secondary antibody with chemiluminescence (Perbio Science UK Ltd, Cheshire, UK) compared to standard molecular weight markers (Invitrogen, Paisley, UK).

\section{Measurement of membrane potential using DIOC5}

MCF-7 cell lines were trypsinized and resuspended in complete medium to a density of $10^{6} / \mathrm{ml}$. The chemosensitizers XR9576 and PSC-833 were used as in the chemosensitivity experiments. The membrane potential sensitive dye DIOC5 was 
added to the cell suspension at a final concentration of $5 \mu \mathrm{M}$. The dye was substituted with media in control untreated samples. After incubating at $37^{\circ} \mathrm{C}$ for $30 \mathrm{~min}$ (with or without XR9576) fluorescence signals using FL1 (525nm, green fluorescence), associated with membrane potential, were measured using the Coulter Epics XL flow cytometer. Each experiment was set up on at least 3 separate occasions. The data were assessed according to the calculated geometric means of the peaks obtained using the WinMDI 2.8 program.

\section{DEP experiments}

The drug resistant and sensitive cells were centrifuged at room temperature at $200 \mathrm{~g}$ for 5 minutes. The pellets were washed and resuspended in isotonic medium consisting of $8.5 \%(\mathrm{w} / \mathrm{v})$ sucrose plus $0.3 \%(\mathrm{w} / \mathrm{v})$ dextrose buffer. The medium conductivity was adjusted to $2.5 \mathrm{mS} / \mathrm{m}$ using PBS and the final conductivity, before use, was verified with a conductivity meter (RS components Ltd, London, UK). The final cell population was counted using a haemocytometer and adjusted to approximately $3 \times 10^{5}$ cells/ $\mathrm{ml}( \pm 15 \%)$. Experiments were repeated 4-6 times with different populations, which were summed prior to modelling. The experimental set up and detailed methodology are described elsewhere [19]. Parameters were derived using a single-shell model and fitted according to the mathematical model described by Broche et al. [ref].

\section{Preparation of cells for fluorescence microscopy:}

Single cell suspensions were prepared as for chemosensitivity testing. Sterile glass cover-slips were placed in sterile tissue culture treated dishes to form a single layer. Single cell suspensions of the various MCF-7 cell lines were pipetted directly onto the 
coverslips in the dishes and allowed to settle for $24 \mathrm{~h}$ in standard tissue culture conditions. Dishes were aspirated dry and then Doxorubicin or daunorubicin containing tissue culture medium at a concentration of $50 \mu \mathrm{M}$ was added to each dish in a volume of $10 \mathrm{ml}$ for 2 hours at $37{ }^{\circ} \mathrm{C}$. These culture conditions have been used previously and 2 hours is sufficient for steady-state levels to be achieved. The drugcontaining medium was then aspirated and the monolayers washed with cold PBS twice. Coverslips were then placed face down onto clean microscope slides and viewed using green fluorescence microscopy at 488nm

\section{Results}

\section{Chemosensitivity Testing:}

Figure 1 depicts the cross-resistance profile for the MCF-7 drug resistant cell lines used in this study alongside Table 1 indicating the $\mathrm{IC}_{50}$ values used to derive the figure. MCF-7DoxR with approximately 5-fold doxorubicin resistance showed a broad cross-resistance to natural product anticancer drugs of varying mechanism of action. Hence, MCF-7DoxR had a cross-resistance profile in keeping with a classical MDR phenotype with moderate levels of drug resistance. The mean $\mathrm{IC}_{50}$ value obtained for MCF-7DoxR with a combination of doxorubicin and PSC-833 was indicated in the footnote to Table 1 . This result was not significantly different from the values obtained with doxorubicin alone $(\mathrm{p}=>0.1)$. We performed additional experiments incorporating XR9576 with the MCF-7DoxR line and found no significant variation from control values (data not shown). There was no evidence for the presence of MDR transporter activity in the MCF-7DoxR cell line based on the chemosensitivity data incorporating MDR modulators as no change in drug sensitivity was seen. In contrast, the MCF-7TaxR cell line with approximately 17-fold resistance 
to paclitaxel showed residual or no cross-resistance to the same natural product anticancer agents. The resistance of MCF-7TaxR to paclitaxel was completely reversed by XR9576 (giving an $\mathrm{IC}_{50}$ value of 4.0nM s.d.+/- 1.5, $\mathrm{p}=<0.02$ ). We can, therefore, attribute the paclitaxel resistance in MCF-7TaxR cells to be entirely due to the presence of P-gp as XR9576 completely reversed the resistance in this cell line variant. The MCF-7MDRI line [20] was shown to be approximately 30-fold resistant to doxorubicin and, hence, was the most drug-resistant cell line in the panel. The doxorubicin resistance was significantly reversed by XR9576, as noted in Table 1.

\section{Detection of MDR-associated proteins}

In addition to the data obtained using the MTT assay, Western immunoblotting (Figure 2) revealed the expression of the MDR protein, P-gp in the resistant breast cancer cell line MCF-7TaxR and MCF-7MDRl lines but not in MCF-7DoxR cells. Moreover, MCF-7TaxR, MCF-7MDRl and MCF-7DoxR were shown to be devoid of MRP1 over-expression and levels of BCRP expression for the entire panel were shown to be very similar.

\section{Assessment of membrane potential using DIOC5}

The corrected peak mean fluorescence of cells incubated with DIOC5 with or without XR9576, relative to the values for MCF-7 parental cells are shown in Table 3. Data shown are representative of several repeat experiments using duplicate or triplicate analyses of each sample. Overall, both the MCF-7 drug resistant variants showed lower membrane potentials than parental cells, even with correction for artefactual transport of DIOC5 by use of XR9576. In line with the chemosensitivity data, it can be seen that XR9576 can modulate DIOC5 accumulation in MCF-7TaxR cells as FL1 
associated fluorescence was shown to increase when this compound was incorporated into the incubation medium. Moreover, the effects of XR9576 on the data obtained for MCF-7DoxR were shown to be marginal, in agreement with the chemosensitivity data (data not shown). Data obtained using PSC-833 with MCF-7DoxR were similarly unaltered

\section{DEP Analysis}

In order to determine the electrical properties of populations, we have counted the number of cells collecting at the electrodes over a period of 1 minute of exposure to a field of a given frequency. This was performed over a range of frequencies from $5 \mathrm{kHz}-20 \mathrm{MHz}$ at 5 frequencies per decade, and modelled using a single-shell model to fit parameters as outlined by Broche et al [ref1]. The fitted parameters were the conductance and capacitance of the membrane, and the conductivity of the cytoplasm, plus a scaling factor; in the frequency window used, it is not possible to obtain accurate data for the cytoplasmic permittivity. Radius data were taken by measuring cell diameters with a microscope and image analysis software. The experiments were repeated four times and the aggregate collection was used. A summary of the data obtained is contained in Table 2. In line with the expression of membrane P-gp, MCF7TaxR cells show an increase in specific membrane capacitance $\left(\mathrm{C}_{\text {spec }}\right)$ suggestive of increases in membrane folding and/or changes in membrane morphology. For MCF7DoxR and MCF-7MDRI there was little variation in this parameter when compared to MCF-7 parental cells. As the other P-gp expressing line in the panel did not show an increase in membrane capacitance it appears that a rise in this parameter is more a consequence of the increased ruffled appearance seen under light microscopy for MCF-7TaxR, rather than an increase in membrane $\mathrm{ABC}$ transporter expression (data 
not shown). The most striking variation in the data was in the cytoplasmic conductivity $(\sigma)$, where the value for MCF-7TaxR is much lower than parental MCF7 cells ( 0.14 and $0.23 \mathrm{~S} / \mathrm{m}$, respectively), whereas MCF-7MDR1 and more so MCF7DoxR cells show a marked increase $(0.4 \mathrm{~S} / \mathrm{m})$ in this parameter. It is possible that changes in viscosity (and hence ion mobility) in cytoplasms with different ionic strengths (in order to maintain osmotic balance) may affect these parameters [ref2]. However, taking cytoplasmic conductivity and plasma membrane potential data together plotted for the 4 individual cell lines in the panel, we can see from Figure 2 that a linear relationship exists between these 2 parameters, with a positive correlation being seen- Pearson's coefficient of correlation $=0.88$, indicating a high likelihood that such effects are relatively small.

\section{Fluorescence microscopy for anthracycline accumulation}

Figure 3 shows anthracyline accumulation for all four cell lines in the panel. Compared with the parental MCF-7 cells, the MCF-7DoxR line shows several areas of groups of cells with weak fluorescence due to defective accumulation. For the MCF-7MDRl cells, the areas of hypo-fluorescent cells are the most apparent for the series. In contrast, the MCF-7TaxR cells are highly fluorescent, with pockets of intense fluorescence. Compared with the picture seen for MCF-7 parental cells, the extent of drug accumulation seen for MCF-7TaxR is very similar. In summary the order of anthracycline accumulation in the cell line panel is MCF-7 $\geq \mathrm{MCF}-7 \mathrm{TaxR}>$ MCF-7DoxR > MCF-7MDR1. 


\section{DISCUSSION}

The majority of MDR cell lines that have been described in the literature over the past 25 years or more possess phenotypes that are likely to be the summation of many drug resistance mechanisms including (but not exclusively) MDR. However, all these models describe a drug resistance mechanism for which defective drug accumulation is the major factor defining that phenotype.

In order to appreciate better the mechanisms underlying the drug resistance profiles of the MCF-7DoxR and MCF-7TaxR lines we undertook a series of biophysical studies using DEP and flow cytometry. The most significant findings of our study relate to cytoplasmic conductivity and also to membrane potential. Here we make the case that P-gp overexpression, as seen in MCF-7TaxR cells, does not appear to influence the level of MDR in those cells, as they are distinctly non- cross-resistant with natural product anticancer agents. The factor that appears to dictate the drug sensitivity / resistance profile in our panel of cells is more closely related to changes in biophysical character that have been assessed using DEP and DIOC5 with flow cytometric analysis. We also show that drug sensitivity, dictated by reduced or low cytoplasmic conductivity, is associated with high levels of cellular drug accumulation, as seen with the anthracyclines.

Plasma membrane potential has frequently been cited as a critical factor underlying anticancer MDR, although there is inconsistency in the literature. We have previously demonstrated flow cytometric studies of membrane potential using DIOC5 with MDR cancer cell lines may produce artefactually low values due to defective accumulation of the dye as a possible consequence of the MDR pump activity, in agreement with 
others $[19 ; 24 ; 25]$. Membrane potential measurements were made in the presence of XR9576, as previous studies have indicated that the membrane potential sensitive dye DIOC5 is a substrate for Pgp-mediated transport [19; 24]. Our data indicate MCF7TaxR as having a lower membrane potential than MCF-7DoxR or MCF-7MDR1, with those for the parental cells lying somewhere in between. Interestingly, MCF7TaxR cells with the lowest membrane potential and lowest cytoplasmic conductivity were overall the most drug sensitive in spite of the presence of membrane P-gp (conferring selective resistance to paclitaxel). An increase in cytoplasmic conductivity could occur as a consequence of increased cytoplasmic molecular transport intracellular trafficking, which is associated with MDR [26; 27].

Here we make the case that P-gp overexpression, as seen in MCF-7TaxR cells, does not appear to influence the level of MDR in those cells, as they are distinctly noncross-resistant with natural product anticancer agents. The factor that appears to dictate the drug sensitivity/resistance profile in our panel of cells is more closely related to changes in biophysical character measured by DEP. A report by Aleman et al. [28] compared MDRl transfected models with drug-selected variants of HeLa. The data indicated that drug selected cells were relatively hyperpolarized when compared to transfected or parental cells, in line with our data obtained for MCF-7DoxR and to some extent for MCF-7MDR1. In contrast, the report by Hoffman et al. [7] indicated a reduced membrane potential and hence a hypopolarised state for a 'pure' $M D R 1$ transfected cell line (i.e. having never been exposed to chemotherapeutic agents). It should be noted that that particular cell line model showed a low, approximately 2fold level of drug resistance and this may have resulted in very subtle changes that may not have been so evident in the data obtained. 
The surface conductance of the plasma membrane indicated by the parameter Ks was shown to be markedly altered (reduced) from parental MCF-7 cells in the MCFMDRI cells, with insignificant changes being seen for the other lines in the panel. MDRl Pgp has been proposed to change lipid-protein interactions on the intramembranous surface of the transporter. This may be brought about by changes in distribution of types of lipids (e.g. glucosylceramide) between the inner and outer leaflets of the plasma membrane giving rise to altered dipole potentials [29]. Such changes may be relevant to our transfected cell line model, but were not apparent for the MCFTaxR Pgp expressing cells.

The membrane capacitance values obtained in our study place non-ABC transporter expressing MCF-7 and MCF-7DoxR (values $12.4 \mathrm{mF} / \mathrm{m}^{2}$ ) as cells with relatively smooth plasma membranes [30] which quotes values of approximately $10 \mathrm{mF} / \mathrm{m}^{2}$. The markedly increased value in capacitance (Cspec) seen for MCF-7TaxR, the smallest cell line in the panel in terms of the cell radius measurements, was also associated with a more noticeably ruffled appearance to its membrane under light microscopy. Reasons for increases in membrane capacitance are known to be membrane folding and exocytotic activity, such as that seen for the white blood cell lineage the eosinophil [31]. It has long been established that a membrane located protein can be expected to influence the capacitance of that membrane. Furthermore, it is known that the function of membraneous protein is regulated by the electrical potential of that membrane. This may be relevant for MCF-7TaxR where we see a hypopolarised state in spite of the presence of P-gp. The influence of a reduced plasma membrane potential and /or the reduced cytoplasmic conductivity (as 
discussed below) in the paclitaxel-resistant cells MCF-7TaxR appeared to modulate the activity of Pgp within these cells so as to render it ineffective in modulating sensitivity and transport of MDR-type substrates, such as doxorubicin, colchicine and VP-16. There is also the possibility that MCF-7TaxR cells express a mutant form of Pgp. However, this is unlikely as the cells were rendered sensitive to MDR modulation and expressed epitopes recognised by antibodies to Pgp.

The technique of DEP provides a measure of the ionic state of cells, indicating the total free (mobile) ionic content of the cytoplasm. Our data indicate a state of low ionic content in MCF-7TaxR cells $(0.14 \mathrm{~S} / \mathrm{m})$ with respect to the parental MCF-7 cells $(0.23 \mathrm{~S} / \mathrm{m})$. Conversely in MCF-7DoxR, the cytoplasmic conductivity was approximately double $(0.4 \mathrm{~S} / \mathrm{m})$ that seen for the parental line $(0.23 \mathrm{~S} / \mathrm{m})$. Other mechanisms underlying MDR have been suggested in the literature, including the protonation and sequestration of drugs in vesicles [26] and the possibility of altered $\mathrm{pH}$ gradients with respect to the parent line [32]. A report by Raghunand et al. [33] described a mathematical model that demonstrates reduced cellular drug accumulation in non-P-gp expressing cells as being due to the activity of an organic cation/H+ antiporter within endosomal membranes. This may form the basis of a number of mechanisms that can result in reduced drug accumulation such as active transport of drug into endosomes (reducing drug levels in the perinuclear region), decreased endosomal $\mathrm{pH}$ and increased plasma membrane $\mathrm{pH}$ gradient. We are uncertain as to the relevance of these mechanisms in our cell line panel, but the increased cytosolic conductivity may be indicative of the processes by which the cytoplasmic $\mathrm{pH}$ is altered. However, as we have not measured the intracellular $\mathrm{pH}$ in our cell line panel we are uncertain as to its role in mediating an MDR phenotype. Moreover, data in the 
literature are somewhat conflicting with regard to the role of intracellular $\mathrm{pH}$ and the MDR phenotype. We propose a model whereby a decrease in ionic content brings about concomitant decrease in trafficking processes, thus facilitating drug accumulation and retention via passive diffusion.

Many chemotherapeutic agents to which MDR cells are resistant are not cationic in nature, but are weakly basic. Hence, it has been argued that changes in membrane potential should not lead to significant changes in the trans-membraneous distribution of those drugs in MDR cells. Paclitaxel itself is not a weakly basic drug as in the case of doxorubicin ( $\mathrm{pKa}$ 8.2) or etoposide (pKa 9.7). It possesses multiple molecular sites with overall no ionisable groups, so is an uncharged molecule. However, it has been reported that an acidic $\mathrm{pH}$ may enhance microtubular stabilization by paclitaxel [34]. Thus, if MCF-7TaxR cells have an alkaline intracellular $\mathrm{pH}$ then this may go some way to explain the basis for their resistance. However, this should also coincide with resistance to colchicine as a more acidic $\mathrm{pH}$ is more favourable for binding of the drug to tubulin [35]. Clearly, this is not the case for MCF-7TaxR, as shown by its lack of cross-resistance to colchicine. The narrow and selective resistance profile of MCF7TaxR, in spite of the presence of P-gp overexpression, may be explained by significant changes in the cytoplasmic nature of the MCF-7TaxR cells, reflected in the low cytoplasmic conductivity. We also accept that there may be other molecular features ascribable to the MCF-7TaxR cell line that result in a cell line that is overall sensitive to many chemotherapeutic agents. However, the current study set out to investigate the biophysics of MDR using this model as a tool to clarify the role of Pgp and other factors in this drug resistance phenotype. 
In conclusion, we propose that there are marked differences in the electrophysiological character between MCF-7 and MDR derivative lines developed under selection pressure, and between the different MDR lines themselves when developed from different anti-cancer drug selection pressure. The cell line models themselves indicate that overexpression of $\mathrm{ABC}$ transporter protein is not necessarily a prerequisite for the MDR phenotype. We propose that marked differences in the nature of the cytoplasm in terms of the conductance measured using DEP go some way towards explaining the relative drug sensitivities of the cell lines. We propose that the technique of DEP may be useful in identifying populations of tumour cells with differing cell sensitivities on the basis of cytoplasmic conductivity measurements. Moreover, the present study highlights the utility of DEP as an effective cell separation technique. 


\section{References}

[1] J.H. Burchenal, E.A.D. Holmburg, The utility of resistant leukaemias in screening for chemotherapeutic activity, Ann NY Acad Sci. 76 (1958) 826-829.

[2] R.M. Wadkins, P.D. Roepe, Biophysical aspects of P-glycoprotein-mediated multidrug resistance, Int Rev Cytol. 171 (1997) 121-165.

[3] P.D. Roepe, L.Y. Wei, J. Cruz, D. Carlson, Lowered membrane potential and altered pHi homeostasis in multidrug resistant (MDR) cells; Further characterisation of a series of MDR cell lines expressing different levels of P-glycoprotein, Biochemistry 32 (1993) 11042-11056.

[4] P.D. Roepe, The role in the MDR protein in altered drug translocation across tumor cell membranes, Biochim Biophys Acta, 1241 (1995) 385-406.

[5] R. Clarke, S. Currier, O. Kaplan, E. Lovelace, V. Boulay, M.M. Gottesman, R.B. Dickson, Effect of P-glycoprotein expression on sensitivity to hormones in MCF-7 human breast cancer cells, J Natl Cancer Inst 84 (1992) 1506-12.

[6] C. Louvet, S. Empereur, D. Fagot, F. Forgue-Lafitte, E. Chastre, A. Zimber, J. Mester, C. Gespach, The arotinoid Ro 40-8757 has antiproliferative effects in drugresistant human colon and breast cancer cell lines in vitro, Cancer Lett 30 (1994) 836. 
[7] M.M. Hoffman, L-Y. Wei, P.D. Roepe, Are altered pHi and membrane potential in hu MDR1 transfectants sufficient to cause MDR protein-mediated multidrug resistance? J.Gen. Physiol. 108 (1996) 295-313.

[8] H. Pohl, Dielectrophoresis. (1978) $1^{\text {st }}$ ed. Cambridge: Cambridge University Press.

[9] T.B. Jones, Electromechanics of Particles, (1995) Cambridge: Cambridge University Press.

[10] M.P. Hughes, Nanoelectromechanics in Engineering and Biology, (2002) $1^{\text {st }}$ ed. Boca Raton: CRC Press.

[11] J.P.H. Burt, R. Pethig, P.R.C. Gascoyne, F.F. Becker, Dielectrophoretic characterization of Friend murine erythroleukaemic cells as a measure of induced differentiation. Biochim Biophys Acta, 1034 (1990) 93-101.

[12] P.R.C. Gascoyne, Y. Huang, R. Pethig, J. Vykoukal, F.F. Becker, Dielectrophoretic separation of mammalian-cells studied by computerized imageanalysis, Meas. Sci. Technol.3 (1992) 439-445.

[13] P.R.C. Gascoyne, R. Pethig, J.P.H. Burt, F.F. Becker, Membrane changes accompanying the induced-differentiation of Friend murine erythroleukemia-cells studied by dielectrophoresis, Biochim Biophys Acta 1149 (1993) 119-126.

[14] P.R.C. Gascoyne, X.B. Wang, Y. Huang, F.F. Becker, Dielectrophoretic separation of cancer cells from blood, IEEE Trans. Ind. Appl. 33 (1997) 670-678. 
[15] F.F. Becker, X.B. Wang, Y. Huang, R. Pethig, J. Vykoukal, P.R.C. Gascoyne, The removal of human leukemia-cells from blood using interdigitated microelectrodes, J Phys D: Appl Phys 27 (1994) 2659-2662.

[16] M. Stephens, M.S. Talary, R. Pethig, A.K. Burnett, K.I. Mills, The dielectrophoresis enrichment of CD34(+) cells from peripheral blood stem cell harvests, Bone Marrow Trans. 18 (1996) 777-782.

[17] X. J. Wang, J. Yang, P.R.C. Gascoyne, Role of peroxide in AC electrical field exposure effects on Friend murine erythroleukemia cells during dielectrophoretic manipulations, Biochim Biophys Acta 1426 (1999) 53-68.

[18] M. Cristofanilli, G. De Gasperis, L.S. Zhang, M.C. Hung, P.R.C. Gascoyne, G.N. Hortobagyi, Automated electrorotation to reveal dielectric variations related to HER2/neu overexpression in MCF-7 sublines, Clin Cancer Res. 8 (2002) 615-619.

[19] F. H. Labeed, H.M. Coley, H. Thomas, M.P. Hughes, Assessment of multidrug resistance reversal using dielectrophoresis and flow cytometry, Biophys J. 85 (2003) 2028-2034.

[20] F. Leonessa, M. Jacobson, B. Boyle, J. Lippman, M. McGarvey, R. Clarke, Effect of tamoxifen on the multidrug resistant phenotype in human breast cancer cells: isobologram, drug accumulation and $\mathrm{M}(\mathrm{r})$ 170,000 glycoprotein (gp170) binding studies, Cancer Res 54 (1994) 441-7. 
[21] T. Mosmann, Rapid colorimetric assay for cellular growth and survivalapplication to proliferation and cytotoxicity assays, J Immunol Meth 65 (1983) 55-63.

[22] P. Mistry, A.J. Stewart, W. Dangerfield, In vitro and in vivo reversal of Pglycoprotein mediated multidrug resistance by a novel potent modulator, XR9576. Cancer Res 61 (2001) 749-758.

[23] T. Yanagisawa, A. Newman, H.M. Coley, J. Renshaw, C.R. Pinkerton, K. Pritchard-Jones, BIRICODAR (VX-710; Incel): an effective chemosensitizer in neuroblastoma. Br J Cancer 80 (1999) 1190-1196.

[24] S. Gollapudi, S. Gupta, Lack of reversal of daunorubicin resistance in HL-60AR cells by cyclosporine A. Anticancer Res 12 (1992) 2127-2132.

[25] B. Vayuvegula, L. Slater, J. Meador, S. Gupta, Correction of altered plasma membrane potentials- a possible mechanism of cyclosporine A and verapamil reversal of pleiotropic drug resistance in neoplasia, Cancer Chemother Pharmacol 22 (1988) 163-168.

[26] Y. Gong, M. Duvvuri, M. Duncan, J.Liu, J.P. Krise, Niemann-Pick C1 protein facilitates the efflux of the anticancer drug daunorubicin from cells according to a novel vesicle mediated pathway, J. Pharmacol Exp Ther 316 (2006) 242-7. 
[27] H. Kipp, I.M. Arias, Intracellular trafficking and regulation of canalicular ATPbinding cassette transporters, Semin Liver Dis 20 (2000) 339-51.

[28] C. Aleman, J-P. Annereau, X-J Liang, C.O. Cardarelli, B. Taylor, J.J. Yin, A. Aszalos, M.M. Gottesman, P-glycoprotein expressed in multidrug resistant cells is not responsible for alterations in membrane fluidity or membrane potential. Cancer Res 63 (2003) 3084-3091.

[29] G.D. Luker, T.P. Flagg, Q. Sha, K.E. Luker, C.M. Pica, C.G. Nichols, D. Piwinica-Worms, MDRl P-glycoprotein reduces influx of substrates without affecting membrane potentials. J Biol Chem 276 (2001) 49053-49060.

[30] R. Pethig, D.B. Kell, The passive electrical properties of biological systems: their significance in physiology, biophysics and biotechnology, Phys Med Biol 32 (1987) 933-970.

[31] S. Scepek, J.R. Coorssen, M. Lindau, 1998. Fusion pore expansion in horse eosinophils is modulated by $\mathrm{Ca}^{2+}$ and protein kinase $\mathrm{C}$ via distinct mechanisms. EMBO J 17 (1998) 4340-4345.

[32] R. Belhoussine, H.Morjani, S. Sharonov, D. Ploton, M. Manfait, Characterisation of intracellular $\mathrm{pH}$ gradients in human multidrug resistant tumour cells by means of 
scanning microspectrofluorometry and dual emission ratio probes. Int J Cancer 81 (1999) 81-89.

[33] N. Raghunand, R. Martinez-Zaguilan, S.H.Wright, R.J. Gillies, pH and drug resistance. II Turnover of acidic vesicles and resistance to weakly basic chemotherapeutic drugs, Biochem Pharmacol 57 (1999) 1047-1058.

[34] I. Ringel, S.B. Horwitz, Effect of alkaline $\mathrm{pH}$ on Taxol-microtubule interactions, J Pharmacol Exp Ther 259 (1991) 855-860.

[35] K. Mukhopadhyay, P.K. Parak. B. Battacharyya, The carboxy terminus of the alpha subunit of tubulin regulates its interaction with colchicine, Biochemistry 29 (1990) 6845-6850.

[ref1] L. Broche, F. H. Labeed, M. P. Hughes. Extraction of dielectric properties for multiple populations from dielectrophoretic collection spectrum data, Phys. Med. Biol. 50 (2005) 2267-2274.

[ref2] J. Gimsa, T. Schnelle, G.Zechel, R. Glaser, Dielectric spectroscopy of human erythrocytes: investigations under the influence of nystatin, Biophys. J. 66 (1994) $1244-1253$ 
TABLE 1

Chemosensitivity testing in MCF-7 cell lines using the MTT assay (continuous drug exposure)

\begin{tabular}{|c|c|c|c|c|}
\hline \multirow{2}{*}{ COMPOUND } & \multicolumn{4}{|c|}{ CELL LINE IC 50 VALUES } \\
\hline & MCF-7 & MCF-7TaxR & MCF-7DoxR & MCF-7MDR-1 \\
\hline Paclitaxel (nM) & $5.3(2.4)$ & $74.4^{*}(18.3)$ & $57.0(26.3)$ & ND \\
\hline Doxorubicin (nM) & $113(40.7)$ & $127.5(38.5)$ & $656^{\#}(199)$ & $3162^{\$}(228)$ \\
\hline Etoposide (uM) & $6.4(5.2)$ & $7.8(3.9)$ & $54.5(12.3)$ & ND \\
\hline Colchicine (nM) & $174(53.5)$ & $258.5(80.5)$ & $878(379)$ & ND \\
\hline
\end{tabular}

Footnote to Table 1: 
Figures shown are the means of IC 50 values for at least 4 separate experiments with the standard deviation shown in parentheses

* Mean IC 50 value obtained for MCF-7TaxR with paclitaxel in combination with $\mathrm{XR} 9576=4.0 \mathrm{nM}(\mathrm{sd}=1.5)$

\# Mean IC 50 value obtained in combination with PSC-833 = 702nM (sd=33)

\$Mean IC 50 value obtained in combination with XR9576=222 nM (s.d. 52) 
TABLE 2

Derived dielectric data for MCF-7 cell lines using a best-fit procedure to match the polarizability spectrum to the observed dielectrophoretic behaviour of particles.

\begin{tabular}{|c|c|cc|}
\hline CELL LINE & $\begin{array}{c}\text { CYTOPLASM } \\
\boldsymbol{\sigma}(\mathbf{S} / \mathbf{m})\end{array}$ & \multicolumn{2}{|c|}{ MEMBRANE } \\
$\boldsymbol{K s}(\mathbf{p S})$ & $\mathbf{C}_{\text {spec }}$ \\
$\left(\mathbf{m F} / \mathbf{m}^{2}\right)$ & $11 \pm 2$ & $12.4 \pm 1.8$ \\
\hline MCF-7 & $0.23 \pm 0.01$ & $10 \pm 2$ & $20.6 \pm 1.1$ \\
\hline MCF-7TaxR & $0.14 \pm 0.01$ & $9 \pm 2$ & $12.4 \pm 0.9$ \\
\hline MCF-7DoxR & $0.40 \pm 0.02$ & $6 \pm 1$ & $12.6 \pm 0.7$ \\
\hline MCF-7/MDR1 & $0.27 \pm 0.02$ & & \\
\hline
\end{tabular}

Figures shown are the means of $>4$ repeat experiments with the limits of best fit indicated.

$\sigma(\mathrm{S} / \mathrm{m})=$ conductivity

$K_{\mathrm{S}}(\mathrm{pS})=$ surface conductance

$$
\mathrm{C}_{\mathrm{spec}}\left(\mathrm{mF} / \mathrm{m}^{2}\right)=\text { capacitance }
$$


TABLE 3

Data showing flow cytometric analysis of MCF-7 cell lines using the membrane potential sensitive dye DIOC5

\begin{tabular}{|c|c|c|c|c|c|}
\hline Cell Line & $\begin{array}{l}\text { DIOC5 } \\
\text { shift } \\
\text { (FL1)* }\end{array}$ & $\begin{array}{c}\text { DIOC5 shift } \\
+ \text { XR9576 }^{\# /} \\
\text { PSC833 }^{\$}\end{array}$ & $\begin{array}{c}\text { Modulation } \\
\text { factor }^{\|}\end{array}$ & $\begin{array}{l}\text { DIOC5 shift } \\
\text { relative to } \\
\text { MCF-7 }\end{array}$ & Interpretation \\
\hline MCF-7 & 100 & $\begin{array}{c}107 / 108 \\
(4 / 15)\end{array}$ & $\begin{array}{l}1.07 \\
(0.04)\end{array}$ & 1.00 & $\begin{array}{c}\text { Control } \\
\text { parental cells } \\
(\mathrm{Pgp}-\mathrm{ve})\end{array}$ \\
\hline MCF-7TaxR & $\begin{array}{l}44 \\
(8.5)\end{array}$ & $\begin{array}{l}69 \# \\
(15)\end{array}$ & $\begin{array}{l}1.51 \\
(0.2)\end{array}$ & 0.64 & $\begin{array}{c}\text { Lowered } \\
\text { membrane } \\
\text { potential }(\mathrm{Pgp}+\mathrm{ve})\end{array}$ \\
\hline MCF-7DoxR & $\begin{array}{l}139 \\
(20.8)\end{array}$ & $\begin{array}{l}137 \$ \\
(31)\end{array}$ & $\begin{array}{l}0.97 \\
(0.07)\end{array}$ & 1.27 & $\begin{array}{l}\text { Increased } \\
\text { membrane } \\
\text { potential } \\
(\text { Pgp-ve) }\end{array}$ \\
\hline MCF-7/MDR 1 & $\begin{array}{l}103 \\
(12.1)\end{array}$ & $\begin{array}{c}135 \# \\
(5)\end{array}$ & $\begin{array}{l}1.31 \\
(0.09)\end{array}$ & 1.25 & $\begin{array}{l}\text { Increased } \\
\text { membrane } \\
\text { potential } \\
(\text { Pgp }+v e)\end{array}$ \\
\hline
\end{tabular}




\section{Footnote to Table 3:}

${ }^{\text {a }}$ Denotes FL1 signal corrected relative to MCF-7 which is set at 100

\# Denotes modulation due to the presence of 100nM XR9576

$\$$ Denotes modulation due to the presence of 4uM PSC-833

"Denotes modulation of fluorescence intensity at FL1 due to modulation calculated as DIOC5 shift in presence of modulator / DIOC5 alone.

Figures in parentheses are the standard deviation for $>3$ repeat experiments. 


\section{Legends to Figures}

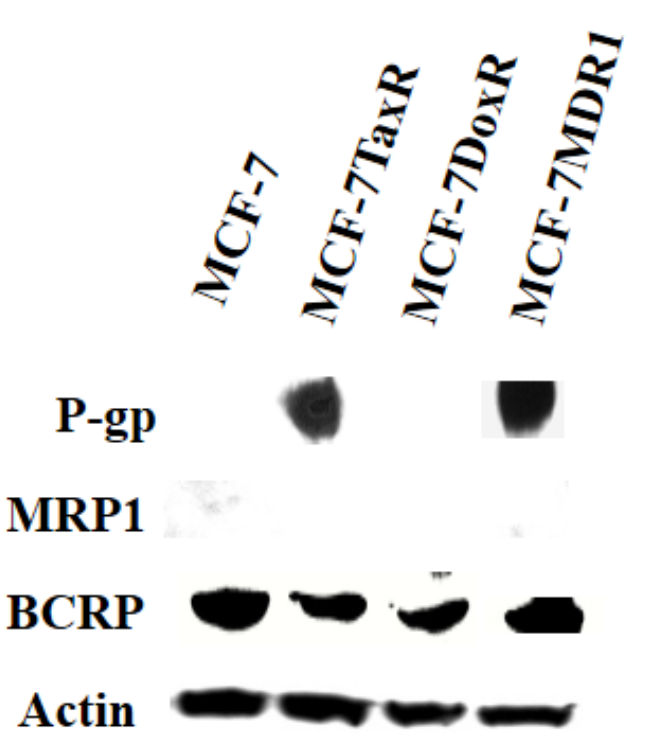

\section{Figure 1.}

Western blot to demonstrate the expression of the MDR proteins (P-glycoprotein) in MCF-7 cell lines. Membrane preparations for each cell line were loaded at a concentration of 40-50 $\mu \mathrm{g}$ of protein onto SDS PAGE $10 \%$ gels with a bis-tris running buffer system. Equivalent loading was checked by stripping the membrane after each Western was run for the individual MDR protein and then re-probed using an antibeta actin antibody (Ab-1) (obtained from CN Biosciences, Beeston, UK). The figure shows reprobing of a blot for beta actin when the blot had been stripped following probing with the primary antibody for MRP. This process was repeated for the other membranes probed with the primary antibodies for P-gp and for BCRP. The positive control for Pgp was the K562AR (doxorubicin-resistant cell line); for BCRP the T8 cell line was used; for MRP the HL-60AR cell line was used (see Methods). 


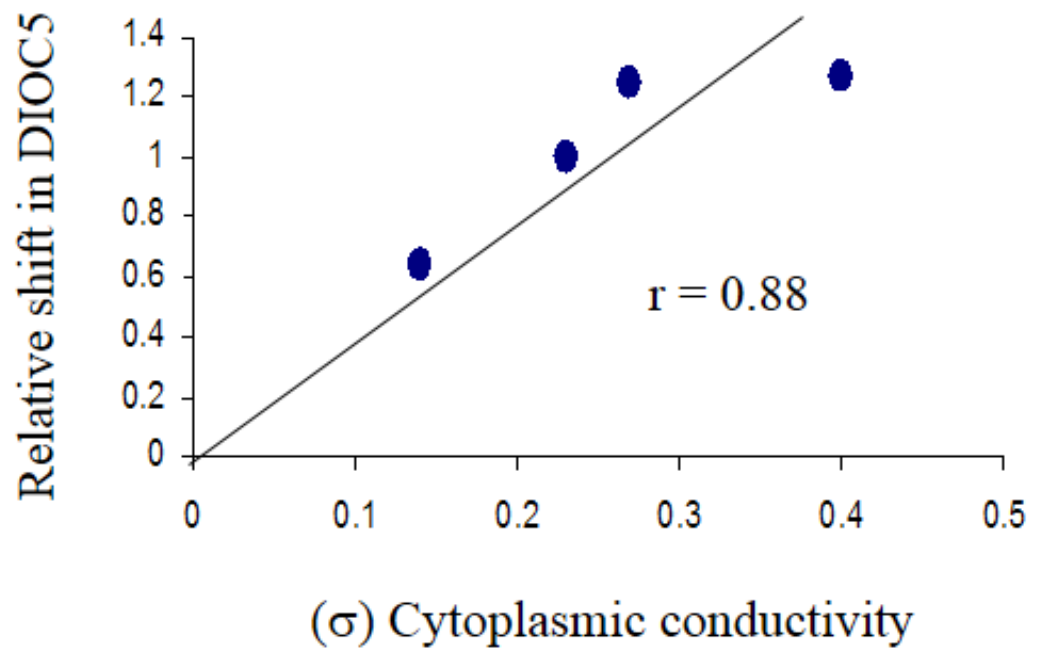

Figure 2.

Plot of DIOC5 shift (y) versus cytoplasmic conductivity (x) showing a linear relationship for the two parameters in MCF-7 cell lines with MDR. Pearson's correlation coefficient $\mathrm{r}=0.88$. 

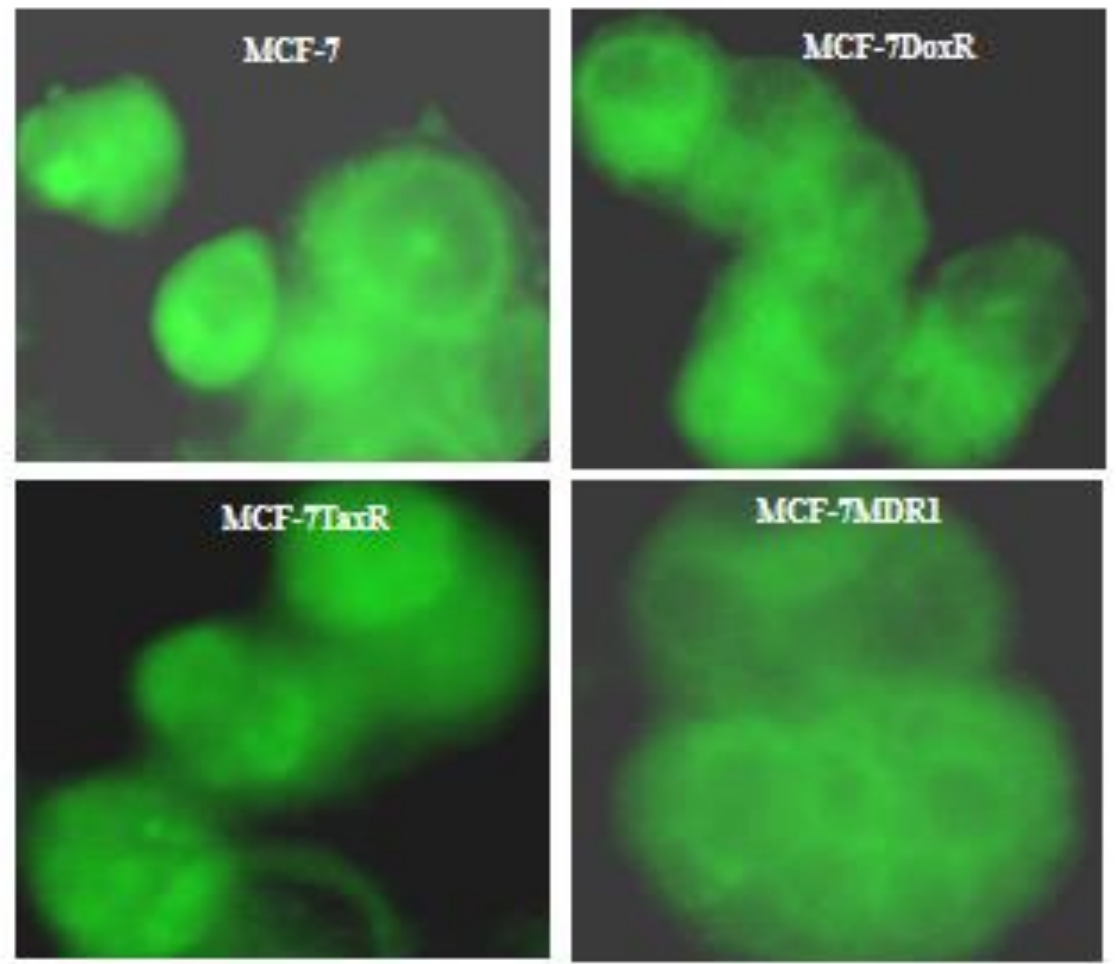

Figure 3.

Fluorescence microscopy images of MCF-7 cell lines following $2 \mathrm{~h}$ incubation with anthracycline. 